\title{
Criminologie
}

\section{Les droits des détenus et leur contrôle : enjeux actuels de la situation canadienne}

\section{Sandra Lehalle}

Volume 40, numéro 2, automne 2007

Peines et pénalité au Canada. Autour des travaux de Pierre Landreville

URI : https://id.erudit.org/iderudit/016856ar

DOI : https://doi.org/10.7202/016856ar

Aller au sommaire du numéro

Éditeur(s)

Les Presses de l'Université de Montréal

ISSN

0316-0041 (imprimé)

1492-1367 (numérique)

Découvrir la revue

Citer cet article

Lehalle, S. (2007). Les droits des détenus et leur contrôle : enjeux actuels de la situation canadienne. Criminologie, 40(2), 127-145.

https://doi.org/10.7202/016856ar
Résumé de l'article

Cet article propose un bref aperçu de l'évolution des droits des personnes détenues au Canada. En dressant le bilan actuel au regard des écrits de Pierre Landreville, nous mettons tout d'abord l'accent sur le cheminement normatif parcouru ainsi que sur les limites rencontrées en termes de ressources nécessaires à la pleine concrétisation des droits des détenus. La seconde partie de cet article analyse les défis contemporains que pose le contrôle du respect des droits des détenus. En se basant sur une recherche empirique récente, nous confrontons l'état d'avancement et l'impact de deux mécanismes de contrôle réclamés dès 1973 par Pierre Landreville : l'Enquêteur correctionnel et le Comité de prévention de la torture des Nations Unies. Nous concluons par une discussion sur l'impact des mécanismes de contrôle et leur utilisation par l'État en tant qu'outil de légitimation.
Ce document est protégé par la loi sur le droit d'auteur. L'utilisation des services d’Érudit (y compris la reproduction) est assujettie à sa politique d'utilisation que vous pouvez consulter en ligne.

https://apropos.erudit.org/fr/usagers/politique-dutilisation/ 


\title{
Les droits des détenus et leur contrôle: enjeux actuels de la situation canadienne
}

\author{
Sandra Lehalle \\ Chercheure post-doctorale \\ Chaire de recherche du Canada en \\ traditions juridiques et rationalité pénale \\ Université d'Ottawa \\ sandra.lehalle@uottawa.ca
}

RÉSUMÉ - Cet article propose un bref aperçu de l'évolution des droits des personnes détenues au Canada. En dressant le bilan actuel au regard des écrits de Pierre Landreville, nous mettons tout d'abord l'accent sur le cheminement normatif parcouru ainsi que sur les limites rencontrées en termes de ressources nécessaires à la pleine concrétisation des droits des détenus. La seconde partie de cet article analyse les défis contemporains que pose le contrôle du respect des droits des détenus. En se basant sur une recherche empirique récente, nous confrontons l'état d'avancement et l'impact de deux mécanismes de contrôle réclamés dès 1973 par Pierre Landreville: l'Enquêteur correctionnel et le Comité de prévention de la torture des Nations Unies. Nous concluons par une discussion sur l'impact des mécanismes de contrôle et leur utilisation par l'État en tant qu'outil de légitimation.

ABSTRACT - This article uses Pierre Landreville's writings as the starting point of a brief historical analysis of the evolution of inmate's rights in Canada. The first section of the article focuses on the normative development of prisoner's rights and the limits it has encountered, particularly with regards to the necessary resources to their full realisation. The second section deals with the ongoing debate concerning the respect of inmates' rights and the oversight mechanisms created to this effect. Using a recent empirical research, we confront the actual state and impact of two oversight mechanisms that Landreville advocated for as early as 1973: the Correctional Investigator and the United Nations sub-committee on prevention of torture. We conclude by discussing the impact of the oversight mechanisms and their legitimating effects. 


\section{Introduction}

S'il existe un domaine des politiques pénales canadiennes qui a connu une évolution notable dans les trente dernières années, il s'agit bien de celui des droits des personnes incarcérées. Sur ce sujet, les écrits de Pierre Landreville de 1973 à 2003 témoignent à la fois du parcours d'un pénologue d'avant-garde et des transformations de la situation des personnes détenues jusqu'aux défis actuels. Si, dans un premier temps, les enjeux ont été essentiellement normatifs, ils dépassent désormais ce cadre pour englober l'ensemble des ressources impliquées dans la mise en application des droits des personnes détenues et inclure la dimension de contrôle du respect effectif de ces droits.

\section{La reconnaissance des droits des détenus: une évolution normative confrontée à ses limites}

Nous pouvons tous croire théoriquement, aux libertés fondamentales, mais il serait tout aussi important, en pratique, de les respecter dans le cas des personnes détenues. (Landreville et Nicolas, 1978: 138)

\section{Des revendications militantes}

La reconnaissance des détenus comme sujets de droit est avant tout le fruit d'un combat militant mené par «quelques hommes de bonne volonté» (Landreville, 1976: 107). Comme le mentionne Landreville (1973), quelques humanistes ont constitué l'exception au désintérêt de la société pour les détenus une fois qu'elle les a rejetés et confiés aux autorités pénitentiaires. Sans remonter aux travaux de John Howard, il suffit de penser au Canada des années 1970 pour prendre la mesure de cette émergence d'un discours militant pour la reconnaissance de droits aux personnes détenues ${ }^{1}$. Au Canada comme ailleurs, des hommes et des femmes se sont impliqués dans cette lutte à travers leur profession d'avocats, de scientifiques ou encore en tant que simples citoyens concernés par le sort de cette population vulnérable que sont les détenus. Organisés en associations comme les sociétés John Howard et Elisabeth Fry, ou encore la Ligue des droits de l'Homme et l'Office des droits des

1. Les années 1960-1970 se sont, en effet, caractérisées, au Canada comme ailleurs, par l'émergence de mouvements sociaux étudiants, ouvriers, nationalistes, féministes, etc., qui ont été essentiels au développement des droits de minorités. 
détenu(e)s, ces citoyens ont contribué à l'émergence de la première étape d'évolution des politiques publiques quelles qu'elles soient: celle du discours des droits des détenus. À cet égard, le rôle des sociologues et criminologues disposant des ressources humaines et matérielles leur permettant de réaliser des recherches dans les établissements de détention fut particulièrement important. À ce titre, Pierre Landreville a été à la fois un acteur, un témoin et un analyste de l'évolution des droits des détenus au Canada durant les trente dernières années. Qu'il s'agisse de réaliser une enquête dans les établissements du Québec en 1972 et 1973 ou encore de s'impliquer dans un front commun pour la fermeture de l'établissement Parthenais entre 1973 et 1975, l'objectif était clairement de «percer ce mur du silence» (Landreville, Gagnon et Desrosiers, 1976: 11).

Au-delà de ce premier objectif de connaissance de la réalité de la détention, apparaît très rapidement un jugement critique face au constat que les personnes détenues sont traitées comme des individus dépourvus de droits. Pour rendre constructive cette critique, les analystes des années 1970 disposaient déjà de l'Ensemble de règles minima pour le traitement des détenus établi par les Nations Unies en 1955 (ONU, 1955). Ces règles, codifiées en 95 articles, avaient pour but d'être adoptées et appliquées par les administrations pénitentiaires des pays signataires, dont le Canada. Parmi ces règles, érigées en «socle minimum du droit pénitentiaire», on trouve le droit à une cellule individuelle, le droit d'information à l'arrivée du détenu, le droit d'exercer des requêtes, etc. N'ayant pas force obligatoire, ces règles ne sont pas, pour autant, un instrument légal mais davantage un guide pour les États qui y souscrivent.

En confrontant la situation concrète des détenus québécois aux règles minima, Landreville (1973) relève des écarts importants et formule diverses recommandations. Il insiste notamment pour que ces règles soient incorporées dans la législation canadienne (qui datait alors de 1962) et mieux connues des services, autorités et groupes intéressés. Il affirme: «[...] nous croyons que l'Ensemble des règles minima devrait être considéré comme la charte des droits de tout individu privé de liberté, qu'il soit inculpé ou non» (Landreville, 1973 :168). Il recommande également de donner aux détenus les moyens de faire valoir leurs droits en créant un organisme national spécialement chargé d'assurer et de contrôler l'application des règles minima. À ce titre, il suggère notamment la création d'un ombudsman correctionnel. 
En arrière-plan de ces revendications concrètes, Landreville (1973) insiste sur le fait que les personnes détenues possèdent tous les droits plus ceux des règles minima. Dans un article publié quelques années plus tard, il affirme clairement: "À mon avis, les détenus doivent conserver tous les droits des autres citoyens sauf celui de circuler dans la communauté» (Landreville, 1976: 108). Si cette affirmation est aujourd'hui une évidence non contestée et un principe consacré, elle témoignait alors d'une position révolutionnaire ou tout au moins minoritaire. Il s'agissait des premiers pas vers la reconnaissance juridique des droits des détenus. Que ce soit à travers des normes internationales ou nationales, l'enjeu de la normalisation juridique semblait à la fois essentiel en termes de ressource pour la cause des détenus et comme produit de cette lutte.

\section{Les normes juridiques: une ressource et un produit de cette reconnaissance}

Le Canada a connu une évolution normative des droits des personnes détenues relativement rapide ${ }^{2}$. Ce développement dont témoignent Lemonde et Landreville (2002) a été marqué par des jalons historiques très prononcés. Parmi ces jalons ${ }^{3}$, on trouve notamment les recours judiciaires qui ont conduit en 1980 la Cour suprême du Canada à reconnaître qu'une personne détenue conserve tous ses droits civils à l'exception de ceux expressément suspendus par la loi ou nécessairement limités par l'incarcération (arrêt Solosky, 1980). La même année, la Cour donne compétence aux tribunaux pour veiller au respect des droits en affirmant que la légalité des décisions prises par les autorités pénitentiaires sera donc dorénavant l'objet d'un contrôle judiciaire (arrêt Martineau, 1980). L'évolution se poursuit ensuite sur le plan législatif. En 1982, la Charte canadienne des droits et libertés intègre à la Constitution toute une série de droits qui ne distinguent aucunement entre les citoyens et s'appliquent donc aux détenus. Dix ans plus tard, en 1992, le législateur consacre officiellement les droits des détenus par une

2. Notamment, si l'on effectue une comparaison avec certains pays européens qui connaissent cette même évolution 20 à 30 ans plus tard.

3. Au-delà des jalons judiciaires détaillés, il est important de souligner l'existence d'autres acteurs et institutions impliqués dans l'évolution normative. Les travaux de nombreuses commissions et comités ont également contribué, à des degrés divers, à l'avancement des droits des détenus; notamment: Archambault (1938), Fauteux (1956), Ouimet (1969), Sauvé (1977), Carson (1984), Archambault (1987) et Arbour (1996). 
réforme majeure de la loi concernant l'incarcération et la libération conditionnelle. Cette consécration normative ${ }^{4}$ vient, entre autres, poser les limites à respecter dans le traitement des détenus des pénitenciers canadiens. Cette loi proclame que les détenus jouissent de tous les droits civils sauf ceux expressément exclus dans la loi; elle formule une liste des droits des détenus et affirme clairement les garanties procédurales de ceux-ci. Ainsi, les normes qui gouvernent les établissements de détention canadiens sont dorénavant ancrées dans la législation.

En matière de droits des détenus, la norme juridique est apparue essentielle à la fois comme produit, comme cadre et comme ressource. Au Canada, le produit de l'action politique en faveur des détenus a été la consécration législative. Dans le processus de reconnaissance des droits des détenus comme dans tout processus politique, les résultats se sont essentiellement articulés autour de l'énoncé de normes juridiques. Le droit a également été utilisé comme cadre ayant la capacité de structurer le contenu des politiques à l'égard des personnes détenues. La norme constitutionnelle ou législative est venue agir comme contrainte qui fixe des balises, des règles du jeu à respecter dans le contenu et dans les processus des politiques correctionnelles. À ce titre, les tribunaux canadiens, et notamment la Cour suprême, ont pleinement donné vie et corps à cette fonction contraignante du droit envers les politiques et les institutions chargées du traitement des détenus. Finalement, le droit a été et continue d'être utilisé comme ressource et outil pour l'action en faveur des droits des personnes détenues. Depuis plus de 40 ans, les divers acteurs concernés par cette question, qu'ils soient détenus ou professionnels, ont usé de la ressource juridique pour se faire entendre et faire valoir leurs intérêts et leurs valeurs. En effet, le droit reste, bien souvent, une ressource privilégiée pour intervenir sur les politiques publiques. Pour les acteurs politiques eux-mêmes, il s'avère être un outil précieux puisqu'il constitue la source de légitimation par excellence de toute action publique (Bernoux, 1985). Ainsi, pour tous les acteurs sociaux impliqués, la ressource juridique est bien un des éléments-clés à mobiliser pour atteindre leurs objectifs, dans ce cas le respect des personnes détenues.

4. Loi régissant le système correctionnel, la mise en liberté sous condition et le maintien en incarcération, et portant création du bureau de l'Enquêteur correctionnel (C-36). 


\section{Les autres ressources en jeu dans la réalisation} des droits des détenus

La norme juridique a donc été et constitue toujours une ressource essentielle pour la protection des «droits des détenus». Cependant, à elle seule, cette ressource peut s'avérer insuffisante, inopérante ou contreproductive, et d'autres ressources sont nécessaires et doivent être mobilisées dans l'implantation de ces droits. En ce sens, la sociologie politique contemporaine nous invite à prendre en compte l'importance et la diversité des ressources qui influencent dès le début les résultats intermédiaires et finaux de la mise en application de toute politique et donc également du développement des droits des détenus. Il est, en effet, possible de confronter les enjeux posés par le développement des droits des personnes détenues à la classification des ressources influençant les processus, les résultats et les effets d'une politique telle qu'elle est élaborée par Knoepfel, Larrue et Varone (2006).

Pour que les droits des personnes détenues prennent véritablement vie, il faut immanquablement prendre en compte la ressource humaine, c'est-à-dire le personnel correctionnel. Que ce soit en termes quantitatifs ou qualitatifs, le développement des droits des détenus fait appel au recrutement et à la formation du personnel qui devra à la fois mettre en œuvre et respecter ces droits. L'actualisation des droits des personnes détenues requiert, en effet, que le personnel soit en nombre suffisant et qu'il soit formé ${ }^{5}$, notamment sur les normes juridiques qui concernent les droits des détenus. Les droits des personnes détenues peuvent se voir limités, voire annihilés par ce manque de ressources: les jours de visites familiales seront réduits en raison du manque de personnel disponible pour les gérer et les surveiller, le processus interne de traitement des plaintes et griefs sera incapable de traiter les plaintes des détenus dans des délais raisonnables, etc. Les besoins en ressources humaines sont souvent étroitement reliés à une autre ressource essentielle: la ressource monétaire. L'argent disponible pour mettre en place la structure, les organismes et les moyens de faire respecter les droits des détenus fait parfois défaut. Qu'il s'agisse de la distribution de documentation juridique, du financement d'aide juridique, de la mise

5. Notons toutefois qu'une formation inadaptée ou défaillante permettrait à certains membres du personnel correctionnel d'adopter des stratégies qui, tout en respectant le cadre de la loi, viendraient cependant brimer les droits des détenus: retard du courrier, contraintes d'accès rapide à un avocat... (Lehalle, 2001). 
en œuvre d'une loi adoptée par le Parlement ou encore du développement et du fonctionnement de mécanismes de contrôle et de recours, le manque de ressources financières est régulièrement argumenté par les autorités politiques et correctionnelles pour expliquer ou justifier les carences ou les délais dans la mise en pratique de certains droits (Lehalle, 2005). Au même titre, les infrastructures des services correctionnels (ou ressource patrimoniale) peuvent s'avérer insuffisantes ou inappropriées pour assurer pleinement le respect des droits proclamés dans les normes correctionnelles nationales ou internationales. Si les établissements de détention sont vétustes et disposent d'un nombre restreint de cellules, de douches et de cours de promenade, il ne sera guère possible de respecter les standards prévus dans les conventions internationales et les législations nationales.

En matière de politique correctionnelle, le temps peut, également, être analysé comme une ressource précieuse. Il est fréquent de constater que l'élaboration et la mise en œuvre d'une nouvelle législation ou politique prennent du temps ${ }^{6}$. La ressource temporelle devient, selon les cas, un outil ou une limite au développement des droits des personnes détenues. Qu'il s'agisse d'accélérer ou de ralentir la réalisation de nouvelles procédures ou pratiques selon des agendas politique et institutionnel ou en raison d'enjeux internes à un éventuel changement de directeur ou de négociations syndicales, le temps est précieusement utilisé par les divers acteurs concernés. Dans le même ordre d'idées, l'information est l'un des fondements de la capacité d'intervention des acteurs. Cette ressource cognitive se constitue, en effet, des connaissances acquises relatives aux données techniques, sociales, économiques et politiques de la question des droits des personnes détenues. Ce savoir fournit une base essentielle aux prises de décision et aux actions entreprises ou non pour favoriser les droits des détenus ${ }^{7}$. L'organisation (ou ressource interactive) qui concerne la qualité de l'organisation administrative et sociale des établissements de détention et des ministères concernés est également mobilisée. Toutes les règles organisationnelles, les interactions entre les divers paliers administratifs sont autant de

6. Il suffit de constater les délais nécessaires à l'avancement de certains projets. Comme nous le verrons plus en avant, il faudra notamment 10 ans pour que l'Enquêteur correctionnel connaisse une consécration législative et plus de 30 ans pour que voie le jour le Sous-comité de prévention de la torture des Nations Unies.

7. À cet égard, les initiatives pour informer les détenus de leurs droits sont particulièrement pertinentes. 
ressources qui peuvent favoriser mais également faire défaut à l'évolution des droits des personnes détenues (Lehalle, 2006).

Finalement, nous trouvons parmi les ressources identifiées par Knoepfel, Larrue et Varone (2006) deux éléments qui semblent particulièrement pertinents pour analyser l'achèvement ou au contraire les carences des droits des détenus. Ces deux ressources sont le soutien politique (ou la ressource majorité) et le consensus (ou la ressource confiance), tous deux des enjeux importants dans l'élaboration et l'exécution des politiques, notamment correctionnelles. Si la ressource majorité fait référence au besoin de faire approuver la politique par la majorité du Parlement, la ressource confiance à trait, elle, à la légitimation et à l'approbation de la politique dans un sens beaucoup plus large qui incorpore les personnes concernées (le personnel et les détenus) mais aussi le public. Au Canada, les grands thèmes des droits des détenus ont fait l'objet d'une loi ayant recueilli la majorité nécessaire pour voir le jour. On constate cependant que les changements de politiques et de pratiques nécessaires pour améliorer la condition actuelle des personnes détenues se heurtent parfois à des difficultés à réunir la ressource confiance et l'approbation nécessaire à leur pleine réalisation ${ }^{8}$. Le manque d'approbation du personnel correctionnel (et parfois même des détenus) peut, en effet, entraver la mise en œuvre quotidienne des normes relatives aux droits des détenus (Vacheret et Lemire, 2006).

Ce rapide parcours des ressources en jeu dans la mise en place de politiques et de pratiques des droits des détenus illustre le fait que la ressource juridique n'est qu'un élément parmi d'autres qui sont tout aussi nécessaires. Les «droits des détenus» ne nécessitent pas uniquement des normes juridiques mais du personnel suffisant et formé, de l'argent et des infrastructures, du temps, des connaissances et une bonne organisation ainsi qu'un appui politique et une certaine approbation. S'il est extrêmement rare de pouvoir réunir toutes les ressources mentionnées, il est souvent primordial de s'assurer de la combinaison de certaines ressources dont l'importance relative sera variable d'une politique à l'autre.

8. La difficulté d'acquérir la confiance du personnel et de l'administration correctionnelle est fréquemment documentée (Enquêteur correctionnel, 1978 et 1996; Lehalle, 2001 et 2006). 


\section{Les limites et revers des normes carcérales}

Si l'arrivée du droit en prison est majoritairement célébrée comme une avancée positive, certaines limites et effets pervers ont rapidement été constatés. Quand on s'attache à dresser le bilan des droits des détenus, on ne peut que constater l'écart existant entre les déclarations de droits des détenus et la réalité vécue par ces derniers. Divers auteurs mentionnent, en effet, qu'il y a souvent loin de la coupe aux lèvres et qu'il existe un monde entre l'affirmation d'un droit et son application quotidienne (Kaminski, 2002; Lemonde, 1995). Il ressort, en effet, que si les droits sont acquis sur papier, la façon dont ils sont appliqués peut poser problème (Lehalle, 2001). Sur ce point, nous suggérons que les explications de cet écart peuvent s'analyser au regard des ressources précédemment mentionnées. L'impact réel des normes juridiques de protection des détenus dépendrait de la mobilisation des diverses ressources qui permettront de leur donner réalité.

Même si elle reste, dans certains domaines, limitée ou imparfaite, cette «colonisation» par le droit a cependant eu des conséquences notables, en particulier sur les rapports sociaux existant dans les établissements de détention. On retrouve, en effet, dans le milieu carcéral, deux manifestations classiques de cette inflation juridique: la judiciarisation et la juridiciarisation des rapports sociaux.

Le milieu carcéral est désormais un milieu où les normes juridiques ont proliféré et se sont diversifiées. Le droit s'est étendu à un nombre croissant de domaines de la vie en détention autrefois gérés de façon informelle. On constate, en effet, une pénétration du droit, de son langage et de sa logique dans des domaines auparavant vides de contenu juridique. Le recours croissant à la norme juridique dans la définition et l'organisation des rapports sociaux, tel qu'il a été analysé dans la société libre par les politologues, se retrouve également au sein des lieux d'incarcération. Cette juridiciarisation des rapports sociaux a été un des fruits de cette reconnaissance des droits des personnes détenues.

Ce fut le cas, également, de l'augmentation du nombre de différends dont la solution est désormais confiée à des institutions judiciaires ou quasi judiciaires. La multiplication des tribunaux administratifs ou toute autre instance compétente pour régler les conflits du milieu carcéral illustre cette tendance de judiciarisation des rapports sociaux. Ce recours de plus en plus fréquent aux tribunaux a pris une dimension particulière depuis l'adoption de la charte qui permet aux détenus de 
contester la validité des normes, politiques et pratiques correctionnelles. Nous reviendrons sur ce point en mentionnant une tentative de déjudiciarisation des différends du milieu correctionnel à travers l'institution d'un ombudsman correctionnel.

Que ce soit par le recours aux normes ou aux tribunaux, la place importante prise par le droit a contribué à la bureaucratisation du milieu carcéral (Jacobs, 1980). Les établissements de détention sont devenus des organisations complexes dans lesquelles les bureaucrates sont les acteurs qui maîtrisent la réglementation et le fonctionnement et finalement une part accrue du pouvoir institutionnel. L'avènement des droits des détenus a contribué à l'évolution des rapports de force entre surveillants et détenus et, notamment, comme le souligne Vacheret (2002), à l'atomisation de chacun de ces deux groupes.

\section{Au-delà des normes juridiques : l'enjeu actuel du contrôle du respect des droits ${ }^{9}$}

Au-delà de la proclamation des droits, nous constatons rapidement que la concrétisation de ceux-ci est parfois plus difficile et peut s'avérer problématique. Sur ce point, Landreville suggérait en 1973 la création d'un ombudsman correctionnel et le renforcement d'un contrôle du respect des standards des Nations Unies. Si la première suggestion a vu le jour très rapidement, la deuxième est actuellement en cours de création.

\section{Le contrôle de l'Enquêteur correctionnel du Canada}

«Il faudrait envisager au fédéral la création d'un ombudsman correctionnel indépendant», affirmaient Dumont et Landreville en 1973. Cette même année, le Canada se dote, en effet, d'un ombudsman spécialisé pour les détenus des pénitenciers fédéraux. L’Enquêteur correctionnel du Canada (EC) fut créé pour s'assurer que les détenus des pénitenciers fédéraux soient traités équitablement ainsi que pour fournir un mécanisme alternatif de règlement des conflits entre ces derniers

9. Les résultats mentionnés dans cette section sont issus d'une recherche réalisée en 2003-2005 sur le contrôle social des établissements de détention. Cette étude qualitative analyse l'ensemble des mécanismes de contrôle du respect des droits des personnes incarcérées en France et au Canada et notamment l'Enquêteur correctionnel et les comités onusiens abordés dans cette section. Pour plus de détails, consulter Lehalle (2006). 
et le Service correctionnel du Canada (SCC). Son mandat comporte clairement deux volets complémentaires. Le premier volet est réactif puisqu'il consiste à recommander des mesures correctives lorsqu'un détenu se trouve lésé dans les droits que lui assurent les législations et les politiques en vigueur. Le second volet vise la prévention des injustices dans le traitement des détenus à travers des recommandations d'application générale sur les pratiques et politiques carcérales.

Après plus de trente années d'existence, une étude ${ }^{10}$ approfondie démontre que cette expérience de déjudiciarisation connaît un succès grandissant en termes de plaintes adressées par les détenus à l'Enquêteur correctionnel (Lehalle, 2005). Dans son action réactive, l'ombudsman semble bien venir pallier certaines limites et carences des recours judiciaires et des systèmes de plaintes internes au Service correctionnel du Canada, souvent surchargés et inadaptés aux situations concrètes de la vie carcérale. En ce qui a trait à son influence sur les pratiques et politiques correctionnelles, l'Enquêteur correctionnel connaît un bilan beaucoup plus mitigé. N'ayant pas de pouvoir de contrainte, il ne peut qu'inciter et tenter de convaincre le Service correctionnel et éventuellement le ministère de donner suite à ses recommandations. Son impact dépend presque exclusivement de la bonne volonté des autorités correctionnelles, qui dépend elle-même de variables aussi diverses que le commissaire en place ou les contingences du moment (Lehalle, 2006).

\section{Le contrôle des Nations Unies sur le Canada}

\section{Le Comité contre la torture (CAT) ${ }^{11}$}

Pour avoir ratifié la Convention contre la torture et autres peines ou traitements cruels, inhumains ou dégradants des Nations Unies le 24 juin 1987, le Canada s'est engagé à respecter les 33 articles de celleci qui visent à prévenir et condamner les actes de torture. Cette convention a instauré un corps de contrôle, le Comité contre la torture

10. Nous avons réalisé en 2005 une analyse qualitative et quantitative du travail de l'Enquêteur correctionnel. L'intégralité des rapports annuels du bureau de l'Enquêteur correctionnel de 1973-2005 a fait l'objet d'une analyse documentaire approfondie. Des observations en milieu fermé et ouvert ont été réalisées en complémentarité de neuf entrevues non directives avec les enquêteurs en poste.

11. Ces résultats découlent d'une analyse documentaire réalisée en 2005 des diverses publications du CAT ainsi que des divers rapports soumis par le gouvernement du Canada (Lehalle, 2005). 
(CAT), dont la mission est de s'assurer que la convention est implantée et observée. Ce comité, composé d'experts, dispose de pouvoirs d'investigation en cas d'allégations de torture, mais son mode de fonctionnement principal consiste à requérir des États membres des rapports périodiques sur les mesures prises pour se conformer aux dispositions de la convention (CAT, 1998). Ainsi, le Canada doit régulièrement exposer tout changement dans sa législation ou ses institutions qui affecte le respect de la convention sur les lieux de sa juridiction, notamment les lieux de détention. Dans ses rapports, le gouvernement doit donc mentionner toute la jurisprudence relevante, toutes les plaintes, requêtes et procédures en cours ainsi que toutes les sentences prononcées pour actes de torture et peines et traitements inhumains et dégradants.

Ainsi, dans les cinq rapports périodiques soumis à ce jour, le Canada présente les mesures adoptées par les gouvernements fédéral et provincial et aborde divers événements ou points problématiques survenus durant la période examinée. Le troisième rapport (Canada, 2000) fait notamment mention des événements survenus à la prison de Kingston en 1994 et les commissions d'enquêtes effectuées, ainsi que l'entrée en vigueur en 1992 de la nouvelle loi qui institue un ombudsman spécialisé pour les détenus: 1'Enquêteur correctionnel ${ }^{12}$. Dans les rapports suivants, le gouvernement canadien fait référence aux travaux parlementaires sur la législation en vigueur, aux mesures prises à la suite du rapport Arbour, à l'ouverture de nouveaux établissements de détention pour femmes, au travail réalisé par l'Enquêteur correctionnel ou encore aux nouvelles politiques disciplinaires et de placement en unité spéciale, ainsi qu'aux stratégies adoptées pour faire face à la surreprésentation et aux besoins des détenus autochtones (Canada, 2002 et 2004). À l'issue de l'examen des derniers rapports, le CAT a fait part de ses préoccupations pour certains sujets particuliers tels les transferts de prisonniers vers les États pratiquant la torture ou encore la fréquence des incidents de violence grave dans les établissements correctionnels fédéraux. Le Comité recommande vivement au Canada de veiller à ce que ses lois respectent le caractère absolu de la Convention (CAT, 2005).

Rapidement, les limites du travail du Comité apparaissent évidentes. En fonctionnant sur la base des rapports des États, le comité est grandement dépendant des informations reçues pour émettre des avis et recom-

12. L'Enquêteur correctionnel existait depuis 1973 mais n'a fait l'objet de dispositions législatives qu'en 1992. 
mandations éclairés. Si l'État fournit des informations partielles ou caduques, les conclusions finales du CAT seront très certainement elles aussi partielles et caduques. Il suffit de mentionner comme exemple les félicitations adressées par le Comité au gouvernement canadien pour l'existence d'une division des droits de la personne au sein du Service correctionnel du Canada à un moment où concrètement cette division se composait d'une seule personne et disposait d'un budget qui ne permettait aucune action substantielle (Lehalle, 2006). De plus, le CAT ne dispose pas de moyens impératifs de forcer les États à suivre ses recommandations. Le caractère préventif de ce contrôle repose principalement sur l'incitation d'aménagement des dispositions de droit interne afin d'empêcher des infractions à la convention (Ponroy et Jacq, 1990).

\section{Le Sous-comité contre la torture}

Après plus de 20 ans de négociations, la création d'un mécanisme de visites régulières des lieux de détention dans le but de prévenir la torture et tout traitement cruel, inhumain ou dégradant, vient finalement de voir le jour à travers l'adoption d'un protocole additionnel à la Convention des Nations Unis. Le Canada a voté pour ce protocole à la fois devant le Conseil économique et social et devant l'assemblée générale. Entré en vigueur le 22 juin 2006, après avoir obtenu les 20 ratifications nécessaires, ce protocole est désormais applicable aux pays qui l'ont signé.

Concrètement, ce protocole établit un système de visites régulières des lieux de détention menées de façon complémentaire entre des organes nationaux et internationaux par des experts indépendants. Il exige, sur le plan national, la mise en place de corps de prévention et, sur le plan onusien, l'établissement d'un sous-comité de prévention de la torture, tous deux faisant l'objet d'autorisations pour visiter tous les établissements de détention des États signataires.

En votant le protocole à la Convention, le Canada s'est engagé à le signer, le ratifier et le respecter. Cependant, la situation canadienne est assez particulière par la nature complexe du système politique en vigueur, qui rend ce processus de ratification plus long et plus compliqué que dans un pays centralisé. Le Canada se trouve dans une situation bien spécifique qui caractérise les États fédéraux tant sur le plan du processus de ratification du protocole que du mécanisme à implanter. En effet, le droit international ne fait pas automatiquement partie du droit national et l'application d'un traité exige, selon la compétence, 
l'intervention du Parlement du Canada et des assemblées législatives des provinces et des territoires. Pour les aspects qui concernent le protocole, nous constatons la division des autorités concernées aussi bien pour l'approbation et l'exécution des traités, la question des droits de l'homme et les domaines de police, de législations criminelles et de lieux de détention.

Il semble bien que, ne disposant pas du pouvoir de forcer les provinces à se conformer aux obligations du protocole, le gouvernement fédéral va s'assurer de leur approbation et coopération avant de signer celui-ci. La structure politique du Canada fait en sorte que la distribution des coûts afférents à l'implantation de ce protocole soit l'objet de discussions et négociations entre le gouvernement fédéral et les gouvernements des provinces et des territoires. En 2005, des négociations sur le protocole étaient en cours mais rendues plus difficiles par la situation minoritaire dans laquelle se trouvait le gouvernement fédéral. Le changement de gouvernement issu des dernières élections fédérales de 2006 est venu encore retarder le processus de signature et de ratification du protocole. Malgré tout, le processus de signature et ratification est en cours. En avril 2006, le gouvernement s'est à nouveau engagé auprès des Nations Unies à considérer ce dossier. Plus concrètement, le Sous-comité des droits internationaux de la personne de la Chambre des communes a décidé en février 2007 d'examiner le protocole, de réaliser des auditions et de proposer une recommandation au Parlement.

Un autre impact de la structure décentralisée du Canada sur le protocole concerne la nature du mécanisme à implanter. Questionné par le CAT à l'issue de l'examen de son dernier rapport, le Canada indique qu'il existe aux paliers fédéral et provincial plusieurs mécanismes de contrôle, tels les tribunaux, les commissions des droits de la personne, l'Enquêteur correctionnel, des organes de déontologie et des ombudsmans. La réponse du Canada au CAT mentionne que des consultations et des analyses sont en cours pour «déterminer si les mécanismes fédéraux et provinciaux actuellement en place au Canada respectent les exigences prévues par le protocole facultatif et, sinon, quelles mesures sont nécessaires pour rencontrer ces exigences» (Canada, 2005). Une des difficultés réside dans la définition large des lieux de détention dont certains ne font pas encore l'objet de contrôle (cellules de détention de la GRC et des polices autochtones par exemple) tandis que d'autres sont soumis à un contrôle dont la conformité aux exigences du protocole 
reste à établir (par exemple des centres de détention d'immigration visités par la Croix-Rouge).

La question se pose, donc, de savoir comment le Canada, qui dispose déjà de mécanismes de contrôle développés, devra procéder à l'évaluation et peut-être au remaniement de ces mécanismes afin de se conformer aux exigences onusiennes. Pour un État fédéré, plusieurs modèles sont possibles: un organisme national unifié ou de multiples organismes qui peuvent être tous régionaux, un fédéral et plusieurs régionaux, ou délimités selon les types de détention, les individus détenus, ou encore une combinaison de l'ensemble de ces possibilités (Pollard, 2005). Une option envisageable serait de donner à l'Enquêteur correctionnel des pouvoirs élargis en termes d'inspection et surtout de juridiction afin que celui-ci soit compétent pour tous les lieux de détention fédéraux et provinciaux.

\section{Le contrôle: une ressource pour l'évolution des droits des personnes détenues?}

Le contrôle, présent ou futur, du respect des droits des détenus canadiens peut très certainement s'analyser comme une avancée progressiste qui contribuera à la concrétisation de ces droits. Cependant, indépendamment du modèle qui sera choisi par le Canada, le ou les contrôles mis en place auront des limitations certaines. Pour convaincre les autorités correctionnelles, il ne disposera pas de pouvoir de contrainte, mais devra miser sur son professionnalisme et ses capacités de conseils, recommandations et persuasion pour asseoir une quelconque influence. Son efficacité sera variable et inégale selon les mécanismes et les personnes impliqués, les thématiques abordées et la collaboration rencontrée de la part de l'administration pénitentiaire, du ministère et du gouvernement concernés. Une étude approfondie de divers mécanismes existants démontrait à ce sujet que les contrôles se heurtent parfois à la résistance paralysante des administrations carcérales et des gouvernements récalcitrants qui ignorent les interpellations et les rapports qui leur sont adressés (Lehalle, 2006). Dans ce rapport d'influence qui s'instaure, l'État remet parfois en cause, indirectement ou publiquement, la crédibilité et l'expertise du mécanisme de contrôle.

L'enjeu du contrôle repose bien souvent sur la capacité d'influencer l'évolution et le changement des politiques et pratiques correctionnelles. Dans bien des cas, un objectif plus ou moins explicite de changement 
anime les mécanismes de contrôle. À travers une mission officielle ou officieuse de prévention et/ou de réaction aux violations des droits des personnes détenues, le contrôle se présente comme une ressource pour faire évoluer le système carcéral à la fois sur le plan pratique et rhétorique. Le rapport contrôle-changement est d'autant plus complexe que, d'une part, le contrôle peut être utilisé stratégiquement par l'État pour légitimer un changement déjà décidé pour des raisons politiques et, d'autre part, la réalisation de certains changements sollicités par le mécanisme de contrôle en place se voit parfois déniée par les autorités de toute relation de causalité avec le travail de celui-ci.

En dépit de ces difficultés, le contrôle, bien que perfectible, incite de diverses façons et par différents moyens l'État, les administrations pénitentiaires et les individus à réduire l'écart existant entre le droit et la réalité carcérale. À la fois concret et symbolique, il contribue à la réflexion et la production de standards sur le «devoir-être» du milieu carcéral en ce qui a trait à ses politiques et ses pratiques, et également à la démocratisation et la transparence des lieux de détention.

\section{Conclusion}

Droits des détenus et mécanismes de contrôle: une nouvelle légitimité pour l'institution carcérale?

Les droits des détenus canadiens ont considérablement évolué depuis les recommandations faites par Landreville en 1973. La reconnaissance normative a été achevée, un ombudsman correctionnel a été mis en place et les Nations Unies sont dans la phase finale d'élaboration d'un contrôle supranational des lieux de détention.

Dans le cas canadien, les mécanismes de contrôle existants (ou à venir) sont directement ou indirectement le fruit de décisions de l'État qui accepte de se soumettre à ce contrôle. Le gouvernement doit, en effet, s'engager à créer et à soumettre ces contrôles supranationaux ou nationaux afin de maintenir la confiance dans l'institution carcérale et ses décideurs. L'enjeu est également de consolider leur légitimité. Finalement, l'État dit qu'il sera contrôlant tout en acceptant d'être contrôlé dans des limites que lui-même stipule. Le contrôle marque donc, à la fois, une limitation de l'État qui est contrôlé et un prolongement de l'État qui est, bien souvent, le maître d'œuvre direct ou indirect de son propre contrôle et finalement l'ultime décideur de l'impact de 
ces contrôles. On constate également que très souvent l'État utilise un ou plusieurs organismes de contrôle pour se justifier et se valider auprès d'un autre organisme de contrôle, ce qui vient encore renforcer ce lien d'interdépendance et de légitimation. Nous avons relevé que le gouvernement canadien se prévaut régulièrement de l'existence de l'Enquêteur correctionnel dans ses rapports aux instances onusiennes.

L'existence et le fonctionnement d'un contrôle du respect des droits des personnes détenues peuvent donc s'analyser, pour l'institution carcérale, comme une boucle de légitimation, telle qu'elle est conçue par Bergeron (1993). Le contrôle constitue bien une nouvelle source de légitimité pour l'État et ses prisons en les faisant paraître plus acceptables et respectables. Concrètement, en contribuant par leur présence et leur travail à l'équilibre entre les personnes privées de liberté et l'institution, les mécanismes de contrôle participent à la survie du système dans son ensemble. En s'assurant que les politiques sont bien exécutées, le contrôle devient possiblement un outil ou un média de légitimation de l'État et de ses prisons.

En étudiant les évolutions normatives, Kaminski (2002) analyse la reconnaissance des droits des détenus comme source potentielle de légitimation de l'institution et de l'État. Il affirme à ce propos: "[C]ette reconnaissance trouve son expression lorsqu'elle est susceptible de produire des effets fonctionnels et symboliques plus rentables pour le système étatique (en l'occurrence pour son administration pénitentiaire)» (Kaminski, 2002: 91). Nous argumentons que le même commentaire peut être formulé sur le développement des mécanismes de contrôle par les États et notamment par le Canada. Le discours du contrôle est issu du discours des droits et de la même idée de limiter l'arbitraire du pouvoir. Par la promotion du contrôle tout comme par la promotion du droit, l'État et les administrations pénitentiaires évitent bien, comme le souligne Kaminski (2002), tout questionnement sur la raison d'être des prisons en privilégiant le débat sur les manières d'être de la prison. À travers le contrôle, l'attention se porte beaucoup plus souvent sur «comment la prison fonctionne ou devrait fonctionner» plutôt que sur «ce qu'elle est ou devrait être». Finalement, si les mécanismes de contrôle s'avèrent être des ressources pour l'évolution du débat, des pratiques et des normes carcérales, ces ressources représentent également pour l'État et ses institutions une source de légitimité nouvelle. 


\section{Références}

Bergeron, P. (1993). L'Etat en fonctionnement. Paris: L'Harmattan.

Bernoux, P. (1985). La sociologie des organisations. Paris: Le Seuil.

Canada (2000). Troisièmes rapports périodiques des États parties devaient présenter en 1996: Canada (19-10-99). Genève: Bureau du Haut Commissaire aux Droits de l'Homme.

Canada (2002). Quatrièmes rapports périodiques des États parties devant être soumis en 2000: Canada (20-08-02). Genève: Bureau du Haut Commissaire aux Droits de l'Homme.

Canada (2004). Cinquièmes rapports périodiques des États parties devant être soumis en 2004: Canada (11-10-2004). Genève: Bureau du Haut Commissaire aux Droits de l'Homme.

Canada (2005). Réponse du Canada à la liste des points: Présentation du $4^{e}$ et $5^{e}$ rapport au CAT. Genève: Bureau du Haut Commissaire aux Droits de l'Homme.

CAT (1998). General guidelines regarding the form and contents of periodic reports to be submitted by states parties. Geneva: Office of the High Commissioner for Human Rights.

CAT (2005). Liste des points à traiter à l'occasion de l'examen des quatrièmes et cinquièmes rapports périodiques du Canada. Genève: Bureau du Haut Commissaire aux Droits de l'Homme.

Dumont, H., \& Landreville, P. (1973). Discipline et droits des détenus dans les institutions pénales au Québec. Revue canadienne de criminologie, 15 (4), 412434.

Jacobs, J. B. (1980). The Prisoner's Right Movement and its Impact, 1960-1980. Crime and Justice, 2, 429-470.

Kaminski, D. (2002). Les droits des détenus au Canada et en Angleterre: entre révolution normative et légitimation de la prison. In O. Schutter \&D. Kaminski, L'institution du droit pénitentiaire: Enjeux de la reconnaissance de droits aux détenus (91-112). Paris: LGDJ.

Knoepfel, P., Larrue, C., \& Varone F. (Ed.) (2006). Analyse et pilotage des politiques publiques. Zurich: Rüegger.

Landreville, P. (1973). L'application des règles minima pour le traitement des détenus au Canada. Acta criminologica, VI, 147-199.

Landreville, P. (1976). Les détenus et les droits de l'homme. Criminologie, 9, 107-117.

Landreville, P., Gagnon, A., \& Derosiers, S. (1976). Les prisons de par ici. Montréal: Éditions Parti pris.

Landreville, P., \& Nicolas, M. (1978). Dans le milieu carcéral: le droit de s'exprimer. Crime et justice, 6, (2), 138-143.

Lehalle, S. (2001). La défense des droits des détenus: Pratiques professionnelles et expériences des avocats en droit carcéral. Montréal: Université de Montréal.

Lehalle, S. (2005). La prison objet de contrôle social: le rôle de l'État et de la société civile en France et au Canada. Revue de Droit Pénal et de Criminologie, $48(2), 1213-1235$. 
Lehalle, S. (2006). Le contrôle social des établissements de détention: les cas de la France et du Canada. Thèse de doctorat. Montréal: Université de Montréal.

Lemonde, L. (1995). L'émergence et l'impact du droit carcéral. Montréal : Université de Montréal.

Lemonde, L., \& Landreville, P. (2002). La reconnaissance des droits fondamentaux des personnes incarcérées: l'expérience canadienne. In O. Schutter \& D. Kaminski, L'institution du droit pénitentiaire. Paris : LGDJ.

ONU (1955). Ensemble de règles minima pour le traitement des détenus. Résolution du premier congrès des Nations Unies pour la prévention du crime et le traitement des délinquants, Genève, 30 août 1955.

Pollard, M. (2005). Implementation of the Optional Protocol to the Convention against Torture and other cruel, Inbuman and Degrading Treatment or Punishment (OPCAT) in federal and other Decentralized States. Paper presented at the seminar OPCAT, Sao Paulo, Brazil, 22-24 June 2005.

Ponroy, E., \& Jacq, C. (1990). Étude comparative des Conventions des Nations Unies et du Conseil de l'Europe relatives à la torture et aux peines ou traitements inhumains ou dégradants. Revue de sciences criminelles et droit pénal comparé, 2.

Vacheret. M. (2002). Relations sociales en milieu carcéral. Une étude des pénitenciers canadiens. Déviance et Société, 1, 83-104.

Vacheret, M., \& Lemire, G. (2006). Anatomie de la prison contemporaine. Montréal: Presses de l'Université de Montréal. 